\title{
ON THE BACKWARD EULER APPROXIMATION OF THE STOCHASTIC ALLEN-CAHN EQUATION
}

\author{
MIHÁLY KOVÁCS, ${ }^{*}$ University of Otago \\ STIG LARSSON, ${ }^{* *}$ Chalmers University of Technology and University of Gothenburg \\ FREDRIK LINDGREN, ${ }^{* *}$ Chalmers University of Technology and University of Gothenburg
}

\begin{abstract}
We consider the stochastic Allen-Cahn equation perturbed by smooth additive Gaussian noise in a spatial domain with smooth boundary in dimension $d \leq 3$, and study the semidiscretization in time of the equation by an implicit Euler method. We show that the method converges pathwise with a rate $O\left(\Delta t^{\gamma}\right)$ for any $\gamma<\frac{1}{2}$. We also prove that the scheme converges uniformly in the strong $L^{p}$-sense but with no rate given.

Keywords: Stochastic partial differential equation; Allen-Cahn equation; additive noise; Wiener process; Euler method; pathwise convergence; strong convergence; factorization method

2010 Mathematics Subject Classification: Primary 60H15
\end{abstract}

Secondary $60 \mathrm{H} 35 ; 65 \mathrm{C} 30$

\section{Introduction}

Let $\mathscr{D} \subset \mathbb{R}^{d}, d \leq 3$, be a spatial domain with smooth boundary $\partial \mathscr{D}$ and consider the stochastic partial differential equation (SPDE) written in the abstract Itô form

$$
\mathrm{d} u+A u \mathrm{~d} t+f(u) \mathrm{d} t=\mathrm{d} W \quad \text { for } t \in(0, T], u(0)=u_{0},
$$

where $\{W(t)\}_{t \geq 0}$ is an $L^{2}(\mathscr{D})$-valued $Q$-Wiener process on a filtered probability space $(\Omega, \mathcal{F}$, $\left.\mathbb{P},\left\{\mathcal{F}_{t}\right\}_{t \geq 0}\right)$ with respect to the normal filtration $\left\{\mathscr{F}_{t}\right\}_{t \geq 0}$. We use the notation $H=L^{2}(\mathscr{D})$ with inner product $\langle\cdot, \cdot\rangle$ and induced norm $\|\cdot\|$ and $V=H_{0}^{1}(\mathcal{D})$. Moreover, $A: V \rightarrow V^{\prime}$ denotes the linear elliptic operator $A u=-\nabla \cdot(\kappa \nabla u)$ for $u \in V$, where $\kappa(x)>\kappa_{0}>0$ is smooth. As usual, we consider the bilinear form $a: V \times V \rightarrow \mathbb{R}$ defined by $a(u, v)=(A u, v)$ for $u, v \in V$, and $(\cdot, \cdot)$ denotes the duality pairing of $V^{\prime}$ and $V$. We denote by $\{E(t)\}_{t \geq 0}$ the analytic semigroup in $H$ generated by the realization of $-A$ in $H$ with $D(A)=H^{2}(\mathscr{D}) \cap H_{0}^{1}(\mathscr{D})$. Finally, $f: \mathscr{D}_{f} \subset H \rightarrow H$ is given by $(f(u))(x)=F^{\prime}(u(x))$, where $F(s)=c\left(s^{2}-\beta^{2}\right)^{2}(c>0)$ is a double well potential. Note that $f$ is only locally Lipschitz and does not satisfy a linear growth condition. It does, however, satisfy a global one-sided Lipschitz condition, which is a key property for proving uniform moment bounds.

We consider a fully implicit backward Euler discretization of (1.1) via the iteration

$$
u^{j}-u^{j-1}+\Delta t A u^{j}+\Delta t f\left(u^{j}\right)=\Delta W^{j} \quad \text { for } j=1,2, \ldots, N, u^{0}=u_{0},
$$

where $\Delta t>0$. Note that this scheme is implicit also in the drift term $f$. In return, the scheme preserves key qualitative aspects of the solution of (1.1) such as moment bounds.

Received 22 November 2013; revision received 18 May 2014.

* Postal address: Department of Mathematics and Statistics, University of Otago, PO Box 56, Dunedin, New Zealand. Email address: mkovacs@maths.otago.ac.nz

** Postal address: Department of Mathematical Sciences, Chalmers University of Technology, SE-412 96 Gothenburg, Sweden. 
The following two results constitute the main results of the paper. For notation, we refer to Section 2. Let $N \in \mathbb{N}, T=N \Delta t$, and $t_{n}=n \Delta t$ for $n=1,2, \ldots, N$. In Theorem 5.1 (pathwise convergence), we show that if $\left\|A^{1 / 2+\varepsilon} Q^{1 / 2}\right\|_{\mathrm{HS}}<\infty$ for some small $\varepsilon>0, \mathbb{E}\left\|u_{0}\right\|_{1}^{2}<\infty$, and $0 \leq \gamma<\frac{1}{2}$, then there are finite random variables $K \geq 0$ and $\Delta t_{0}>0$ such that, a.s.,

$$
\sup _{t_{n} \in[0, T]}\left\|u\left(t_{n}\right)-u^{n}\right\| \leq K \Delta t^{\gamma}, \quad \Delta t \leq \Delta t_{0}
$$

In Theorem 5.2 (strong convergence), we prove that if, in addition to $\left\|A^{1 / 2+\varepsilon} Q^{1 / 2}\right\|_{\mathrm{HS}}<\infty$ for some small $\varepsilon>0$, we have $p \geq 1$ and $\mathbb{E}\left\|u_{0}\right\|_{1}^{q}<\infty$ for $q>p, q \geq 2$, then

$$
\lim _{\Delta t \rightarrow 0} \mathbb{E} \sup _{t_{n} \in[0, T]}\left\|u\left(t_{n}\right)-u^{n}\right\|^{p}=0 .
$$

Since the method of proof uses a priori bounds obtained via energy arguments together with a pathwise error analysis based on the mild formulation of the equation, a strong rate cannot be obtained via this line of argument. We would like to point out that the strong convergence of the backward Euler scheme is somewhat surprising given the superlinearly growing character of $f$; see also the discussion in [14]. The authors are not aware of any results where strong convergence results, with or without rates, have been obtained for a timediscretization scheme for an SPDE with nonglobal Lipschitz nonlinearity without linear growth (for stochastic ordinary differential equations, we refer the reader to [13]). There are many results on pathwise and strong convergence of the backward Euler scheme (usually explicit in the drift term $f$ ) under global Lipschitz conditions (or local Lipschitz with linear growth conditions); see, for example, [5] and [9]-[12] and the references therein. Gyöngy and Millet [10] also considered monotone nonglobal Lipschitz nonlinearity but with finite-dimensional noise. For nonglobal Lipschitz nonlinearities the relatively recent method developed in [14] uses a scheme which is based on the mild formulation of the SPDE. This is also employed, for example, in [3]. In that setting pathwise error estimates are derived but strong convergence results would be rather difficult to obtain as the method loses the information about the onesided Lipschitz condition on $f$, which can only be exploited in a variational or weak solution approach. We also mention Printems [25] where convergence in probability is obtained without global Lipschitz conditions for the backward Euler scheme.

Spatial pathwise convergence results for certain semilinear SPDEs with nonglobal Lipschitz $f$ without linear growth are obtained in [2] and [3], both using a spectral Galerkin approximation. Concerning spatial strong convergence the authors are only aware of [19] and [26], both with rates, based on a spectral Galerkin method and a finite difference method, respectively. In the latter two papers the authors use energy type arguments, and, hence, they can fully exploit the one-sided Lipschitz character of $f$. In [1] Alabert and Gyöngy obtained pathwise convergence rates for a finite difference spatial semidiscretization of a white-noise driven stochastic Burgers' equation in one spatial dimension.

Finally, we would like to note that (1.2) is also referred to as Rothe's method. Since we can prove both pathwise and strong convergence, we can set up a nonlinear wavelet-based adaptive algorithm to solve the elliptic equation in each time-step and obtain an implementable scheme, which converges both pathwise and strongly in a similar way as in [4] and [17].

This paper is organized as follows. In Section 2 we collect frequently used results from infinite dimensional analysis and introduce some notation. In Section 3 we discuss the spatial Sobolev regularity of the solution and the Hölder regularity in time. In Section 4 we prove maximal type $p$ th moment bounds on $u^{n}$ (Propositions 4.1 and 4.2), which are in fact the exact 
analogues of the ones on $u(t)$ (Proposition 3.1). Here, we highlight that for $p=2$ the bounds only grow linearly in $T$, while for $p>2$ they grow exponentially because of a Gronwall argument. In Section 5 we state and prove the main results of this paper, on the convergence of (1.2). An important part of the proof is a maximal type error estimate for the linear part (Proposition 5.1), where we employ a discrete version of the celebrated factorization method.

\section{Preliminaries}

Throughout this paper we will use various norms for linear operators on a Hilbert space. We denote by $\mathcal{L}(H)$, the space of bounded linear operators on $H$ with the usual operator norm denoted by $\|\cdot\|$. If for a positive semidefinite operator $T: H \rightarrow H$, the sum

$$
\operatorname{Tr} T:=\sum_{k=1}^{\infty}\left\langle T e_{k}, e_{k}\right\rangle<\infty
$$

for an orthonormal basis (ONB) $\left\{e_{k}\right\}_{k \in \mathbb{N}}$ of $H$, then we say that $T$ is trace-class. In this case $\operatorname{Tr} T$, the trace of $T$, is independent of the choice of the ONB. If for an operator $T: H \rightarrow H$, the sum

$$
\|T\|_{\mathrm{HS}}^{2}:=\sum_{k=1}^{\infty}\left\|T e_{k}\right\|^{2}<\infty
$$

for an ONB $\left\{e_{k}\right\}_{k \in \mathbb{N}}$ of $H$, then we say that $T$ is Hilbert-Schmidt and call $\|T\|_{\mathrm{HS}}$ the HilbertSchmidt norm of $T$. The Hilbert-Schmidt norm of $T$ is independent of the choice of the ONB. We have the following well-known properties of the trace and Hilbert-Schmidt norms; see, for example, [7, Appendix C],

$$
\begin{gathered}
\|T\| \leq\|T\|_{\mathrm{HS}}, \quad\|T S\|_{\mathrm{HS}} \leq\|T\|_{\mathrm{HS}}\|S\|, \quad\|S T\|_{\mathrm{HS}} \leq\|S\|\|T\|_{\mathrm{HS}}, \\
\operatorname{Tr} Q=\left\|Q^{1 / 2}\right\|_{\mathrm{HS}}^{2}=\|T\|_{\mathrm{HS}}^{2}=\left\|T^{*}\right\|_{\mathrm{HS}}^{2}, \quad \text { if } Q=T T^{*} .
\end{gathered}
$$

Next, we introduce fractional order spaces and norms. It is well known that our assumptions on $A$ and on the spatial domain $\mathscr{D}$ imply the existence of a sequence of nondecreasing positive real numbers $\left\{\lambda_{k}\right\}_{k \geq 1}$ and an orthonormal basis $\left\{e_{k}\right\}_{k \geq 1}$ of $H$ such that

$$
A e_{k}=\lambda_{k} e_{k}, \quad \lim _{k \rightarrow+\infty} \lambda_{k}=+\infty .
$$

Using the spectral functional calculus for $A$, we introduce the fractional powers $A^{s}, s \in \mathbb{R}$, of $A$ as

$$
A^{s} v=\sum_{k=1}^{\infty} \lambda_{k}^{s}\left(v, e_{k}\right) e_{k}, \quad D\left(A^{s}\right)=\left\{v \in H:\left\|A^{s} v\right\|^{2}=\sum_{k=1}^{\infty} \lambda_{k}^{2 s}\left(v, e_{k}\right)^{2}<\infty\right\}
$$

and spaces $\dot{H}^{\beta}=D\left(A^{\beta / 2}\right)$ with inner product $\langle u, v\rangle_{\beta}=\left\langle A^{\beta / 2} u, A^{\beta / 2} v\right\rangle$ and induced norms $\|v\|_{\beta}=\left\|A^{\beta / 2} v\right\|$. It is well-known that if $0 \leq \beta<\frac{1}{2}$ then $\dot{H}^{\beta}=H^{\beta}$ and if $\frac{1}{2}<\beta \leq 2$ then $\dot{H}^{\beta}=\left\{u \in H^{\beta}:\left.u\right|_{\partial \mathcal{D}}=0\right\}$, where $H^{\beta}$ denotes the standard Sobolev space of order $\beta$.

We recall the fact that the semigroup $\{E(t)\}_{t \geq 0}$ generated by $-A$ is analytic and therefore it follows from [23, Theorem 6.13] that for $t>s>0$,

$$
\begin{gathered}
\left\|A^{\beta} E(t) v\right\| \leq C t^{-\beta}\|v\|, \quad \beta \geq 0, \\
\left\|A^{\beta}(E(t)-E(s)) v\right\| \leq C s^{-(\beta+\gamma)}|t-s|^{\gamma+\rho}\left\|A^{\rho} v\right\|, \quad \beta \geq 0,0 \leq \gamma+\rho \leq 1 .
\end{gathered}
$$


We will also use Itô's isometry and the Burkholder-Davies-Gundy inequality for Itô integrals of the form $\int_{0}^{t}\langle\eta(s), \mathrm{d} \tilde{W}(s)\rangle$, where $\tilde{W}$ is a $\tilde{Q}$-Wiener process. For this kind of integral, Itô's isometry, [7, Proposition 4.5] reads as

$$
\mathbb{E}\left|\int_{0}^{t}\langle\eta(s), \mathrm{d} \tilde{W}(s)\rangle\right|^{2}=\mathbb{E} \int_{0}^{t}\left\|\tilde{Q}^{1 / 2} \eta(s)\right\|^{2} \mathrm{~d} s,
$$

and the Burkholder-Davies-Gundy inequality, [7, Lemma 7.2], takes the form

$$
\mathbb{E} \sup _{t \in\left[0, t_{0}\right]}\left|\int_{0}^{t}\langle\eta(s), \mathrm{d} \tilde{W}(s)\rangle\right|^{p} \leq C_{p} \mathbb{E}\left\{\int_{0}^{t_{0}}\left\|\tilde{Q}^{1 / 2} \eta(s)\right\|^{2} \mathrm{~d} s\right\}^{p / 2} \text { for } p \geq 2 .
$$

Finally, if $Y$ is an $H$-valued Gaussian random variable with covariance operator $\tilde{Q}$ then by [7, Corollary 2.17], we can bound its $p$ th moments via its covariance operator as

$$
\mathbb{E}\|Y\|^{2 p} \leq C_{p}\left(\mathbb{E}\|Y\|^{2}\right)^{p}=C_{p}(\operatorname{Tr} \tilde{Q})^{p}=\left\|\tilde{Q}^{1 / 2}\right\|_{\mathrm{HS}}^{2 p} .
$$

\section{Regularity of the solution}

The following existence, uniqueness, and regularity result can essentially be found in [21, Example 3.5] for $\mathscr{D}=[0,1]$, where it is stated with ess sup instead of sup for the second term in (3.1). It is remarked there, [21, Remark 3.4], that the result can be proved in higher dimensions by using [22, Example 3.2], where domains with smooth boundary are considered. Finally, by [20, Theorem 1.1], the ess sup can be replaced by sup in the second term as stated below in (3.1). We also note that for the equation considered in this paper, this result can be obtained by using the deterministic Ljapunov functional $J(u)=\|\nabla u\|_{1}^{2}+\int_{\mathscr{D}} F(u) \mathrm{d} x$ and Itô's formula in a way analogous to [16, Theorem 3.1 and Corollary 3.2]; see also [6].

For the definition of variational solution; see [24, Definition 4.2.1].

Proposition 3.1. If $\left\|A^{1 / 2} Q^{1 / 2}\right\|_{\mathrm{HS}}<\infty$ and $\mathbb{E}\left\|u_{0}\right\|_{1}^{p}<\infty$ for some $p \geq 2$, then there is a unique variational solution u of (1.1). Furthermore, there is $C_{T}>0$ such that

$$
\mathbb{E} \sup _{t \in[0, T]}\|u(t)\|^{p}+\mathbb{E} \sup _{t \in[0, T]}\|u(t)\|_{1}^{p} \leq C_{T} .
$$

In this case, $u$ is also a mild solution; see [24, Proposition F.0.5 and Remark F.0.6]; i.e. $u$ satisfies the integral equation

$$
u(t)=E(t) u_{0}+\int_{0}^{t} E(t-s) f(u(s)) \mathrm{d} s+W_{A}(t), \quad t \in[0, T],
$$

a.s., where the stochastic convolution $W_{A}$ is defined by

$$
W_{A}(t)=\int_{0}^{t} E(t-s) \mathrm{d} W(s) .
$$

This ultimately follows from the fact that the noise is additive trace class and that, by Sobolev's inequality,

$$
\|f(u(t))\| \leq C\left(\|u(t)\|+\|u(t)\|_{L^{6}}^{3}\right) \leq C\left(\|u(t)\|+\|u(t)\|_{1}^{3}\right),
$$

which is bounded a.s. for $t \in[0, T]$ by Proposition 3.1. Note that here, in order to be able to use Sobolev's inequality, it is crucial that $d \leq 3$ and that the nonlinearity $f$ is at most cubic.

Next, we look at the pathwise Hölder regularity of $u$. First, we consider the stochastic convolution $W_{A}$. 
Lemma 3.1. Let $0<\beta \leq 1,\left\|A^{(\beta-1) / 2} Q^{1 / 2}\right\|_{\mathrm{HS}}<\infty$, and $p>2 / \beta$. Then there is $a$ nonnegative real random variable $K$ with $\mathbb{E} K^{p}<\infty$ such that, a.s.,

$$
\sup _{t \neq s \in[0, T]} \frac{\left\|W_{A}(t)-W_{A}(s)\right\|}{|t-s|^{\gamma}} \leq K \quad \text { for } 0 \leq \gamma<\frac{(\beta p / 2)-1}{p} .
$$

Proof. Let $t>s \geq 0$. Note that the stochastic integrals below are Gaussian random variables and, hence, we can use (2.7) to bound their $p$ th moments. Therefore,

$$
\begin{aligned}
\mathbb{E}\left\|W_{A}(t)-W_{A}(s)\right\|^{p \leq} & C_{p} \mathbb{E}\left\|\int_{s}^{t} E(t-\sigma) \mathrm{d} W(\sigma)\right\|^{p} \\
& +C_{p} \mathbb{E}\left\|\int_{0}^{s} E(t-\sigma)-E(s-\sigma) \mathrm{d} W(\sigma)\right\|^{p} \\
\leq & C_{p}\left(\int_{s}^{t}\left\|E(t-\sigma) Q^{1 / 2}\right\|_{\mathrm{HS}}^{2} \mathrm{~d} \sigma\right)^{p / 2} \\
& +C_{p}\left(\int_{0}^{s}\left\|E(t-\sigma)-E(s-\sigma) Q^{1 / 2}\right\|_{\mathrm{HS}}^{2} \mathrm{~d} \sigma\right)^{p / 2} \\
\leq & C|t-s|^{\beta p / 2},
\end{aligned}
$$

where the last inequality is shown in the proof of [17, Theorem 4.2]. Then the statement follows from Kolmogorov's criterion; see, for example, [18, Theorem 1.4.1].

With the above preparations, we now prove the Hölder continuity of $u$. Note that the result is suboptimal compared to the corresponding result for $W_{A}$ in Lemma 3.1, which requires only $\beta=1$ to obtain the same Hölder exponent, while here we assume that $\beta=2$. This is a consequence of the fact that we use the mild formulation here and, hence, cannot exploit the one-sided Lipschitz condition on $f$ but only its cubic growth.

Proposition 3.2. Let $\left\|A^{1 / 2} Q^{1 / 2}\right\|_{\mathrm{HS}}<\infty, \mathbb{E}\left\|u_{0}\right\|_{1}^{2}<\infty$ and $T>0$. Then for all $\gamma \in\left[0, \frac{1}{2}\right)$ there is a finite nonnegative random variable $K$ such that, a.s.,

$$
\sup _{t \neq s \in[0, T]} \frac{\|u(t)-u(s)\|}{|t-s|^{\gamma}} \leq K .
$$

Proof. Let $T>0,0 \leq s<t \leq T$, and $0 \leq \gamma<\frac{1}{2}$. We use the mild formulation (3.2) to represent $u(t)-u(s)$ as follows:

$$
\begin{aligned}
u(t)-u(s)= & (E(t)-E(s)) u_{0}+\int_{s}^{t} E(t-r) f(u(r)) \mathrm{d} r \\
& +\int_{0}^{s}\left(E(t-r)-E(s-r) f(u(r)) \mathrm{d} r+W_{A}(t)-W_{A}(s) .\right.
\end{aligned}
$$

The estimate in (2.4) with $\beta=\gamma=0$ and $\rho=\frac{1}{2}$ implies that $\left\|(E(t)-E(s)) u_{0}\right\| \leq C \mid t-$ $\left.s\right|^{1 / 2}\left\|u_{0}\right\|_{1}$. The second term can be bounded, using Proposition 3.1 and (3.3),

$$
\begin{aligned}
\left\|\int_{s}^{t} E(t-r) f(u(r))\right\| & \leq \int_{s}^{t}\|E(t-r)\|\|f(u(r))\| \mathrm{d} r \\
& \leq C|t-s| \sup _{r \in[0, T]}\left(\|u(r)\|+\|u(r)\|_{1}^{3}\right) \leq K|t-s| .
\end{aligned}
$$


In a similar fashion, using this time (2.4) with $\beta=\rho=0$ and $\frac{1}{2} \leq \gamma<1$,

$$
\begin{aligned}
& \left\|\int_{0}^{s}(E(t-r)-E(s-r)) f(u(r)) \mathrm{d} r\right\| \\
& \quad \leq \int_{0}^{s}\|(E(t-r)-E(s-r))\| \mathrm{d} r \sup _{r \in[0, T]}\left(\|u(r)\|+\|u(r)\|_{1}^{3}\right) \\
& \quad \leq K|t-s|^{\gamma} \int_{0}^{s} r^{-\gamma} \mathrm{d} r \\
& \quad \leq K T^{1-\gamma}|t-s|^{\gamma} .
\end{aligned}
$$

Finally, we note that $\left\|Q^{1 / 2}\right\|_{\mathrm{HS}} \leq C\left\|A^{1 / 2} Q^{1 / 2}\right\|_{\mathrm{HS}}<\infty$ by $(2.1)$ as $A^{-1 / 2} \in \mathcal{L}(H)$ so that we can use Lemma 3.1 with $\beta=1$ to conclude the proof.

\section{A priori moment bounds}

Our first result bounds the second moment of the Euler iterates in (1.2). The proof uses a kind of bootstrapping argument and as a result we avoid Gronwall's lemma. Therefore, we are able to obtain bounds that only grow linearly with $T$ instead of exponentially. Since these bounds will be used in the Gronwall step in the pathwise convergence analysis, the constants appearing there will grow exponentially with time instead of double-exponentially. We have to use test functions in the energy arguments below that are different from the ones used in the deterministic setting, for example, in [8], because of the presence of a nondifferentiable right-hand side. This ultimately forces us to choose a scheme that is also implicit in the drift in order to be able to use the one-sided Lipschitz property of $f$.

Proposition 4.1. Let $I_{N}=\{1,2, \ldots, N\}$ and $T=N \Delta t$. If $\left\|A^{1 / 2} Q^{1 / 2}\right\|_{\mathrm{HS}}<\infty$ and $\mathbb{E}\left\|u_{0}\right\|_{1}^{2}<\infty$, then there is $C>0$ independent of $T$ such that

$$
\mathbb{E} \sup _{l \in I_{N}}\left\|u^{l}\right\|^{2}+\mathbb{E} \sup _{l \in I_{N}}\left\|u^{l}\right\|_{1}^{2} \leq C(1+T) .
$$

Proof. First note that it is enough to bound the second term on the left-hand side since $\|\cdot\| \leq C\|\cdot\|_{1}$. Taking the inner product of (1.2) with $u^{j}$, we obtain

$$
\left\langle u^{j}-u^{j-1}, u^{j}\right\rangle+\Delta t\left\|u^{j}\right\|_{1}^{2}+\Delta t\left\langle f\left(u^{j}\right), u^{j}\right\rangle=\left\langle\Delta W^{j}, u^{j}\right\rangle .
$$

Using the identity $\langle x-y, x\rangle=\frac{1}{2}\left(\|x\|^{2}-\|y\|^{2}\right)+\frac{1}{2}\|x-y\|^{2}$ and the fact that for some $C>0$, we have $s f(s) \geq-C$ for all $s \in \mathbb{R}$, we obtain

$$
\begin{array}{r}
\frac{1}{2}\left(\left\|u^{j}\right\|^{2}-\left\|u^{j-1}\right\|^{2}\right)+\frac{1}{2}\left\|u^{j}-u^{j-1}\right\|^{2}+\Delta t\left\|u^{j}\right\|_{1}^{2} \\
\leq C \Delta t+\left\langle\Delta W^{j}, u^{j}-u^{j-1}\right\rangle+\left\langle\Delta W^{j}, u^{j-1}\right\rangle .
\end{array}
$$

Using a kick back argument on the second term on the right and summing from 1 to $n(1 \leq$ $n \leq N)$ it follows that

$$
\begin{aligned}
\left\|u^{n}\right\|^{2} & +\sum_{j=1}^{n}\left\|u^{j}-u^{j-1}\right\|^{2}+\Delta t \sum_{j=1}^{n}\left\|u^{j}\right\|_{1}^{2} \\
& \leq C\left(T+\left\|u_{0}\right\|^{2}+\sum_{j=1}^{n}\left(\left\|\Delta W^{j}\right\|^{2}+\left\langle\Delta W^{j}, u^{j-1}\right\rangle\right)\right) .
\end{aligned}
$$


Taking expectation, using the fact that $\Delta W^{j}$ is Gaussian with covariance operator $\Delta t Q$ and, hence, $\mathbb{E}\left\|\Delta W^{j}\right\|^{2}=\Delta t \operatorname{Tr} Q=\Delta t\left\|Q^{1 / 2}\right\|_{\mathrm{HS}}^{2}$, and that $\mathbb{E} \sum_{j=1}^{n}\left\langle\Delta W^{j}, u^{j-1}\right\rangle=0$, we conclude,

$$
\mathbb{E}\left\{\left\|u^{n}\right\|^{2}+\sum_{j=1}^{n}\left\|u^{j}-u^{j-1}\right\|^{2}+\Delta t \sum_{j=1}^{n}\left\|u^{j}\right\|_{1}^{2}\right\} \leq C\left(T+\mathbb{E}\left\|u_{0}\right\|^{2}+T\left\|Q^{1 / 2}\right\|_{\mathrm{HS}}^{2}\right) .
$$

Next, we take the inner product of (1.2) with $A u^{j}$ and obtain, similarly as above,

$$
\frac{1}{2}\left(\left\|u^{j}\right\|_{1}^{2}-\left\|u^{j-1}\right\|_{1}^{2}\right)+\frac{1}{2}\left\|u^{j}-u^{j-1}\right\|_{1}^{2}+\Delta t\left\|u^{j}\right\|_{2}^{2}+\Delta t\left\langle f\left(u^{j}\right), u^{j}\right\rangle_{1}=\left\langle\Delta W^{j}, u^{j}\right\rangle_{1} .
$$

Since $f^{\prime}(s) \geq-C$, we have

$$
\left\langle f\left(u^{j}\right), u^{j}\right\rangle_{1}=\left\langle\nabla f\left(u^{j}\right), \nabla u^{j}\right\rangle=\left\langle f^{\prime}\left(u^{j}\right) \nabla u^{j}, \nabla u^{j}\right\rangle \geq-C\left\|u^{j}\right\|_{1}^{2} .
$$

Hence,

$$
\begin{aligned}
& \frac{1}{2}\left(\left\|u^{j}\right\|_{1}^{2}-\left\|u^{j-1}\right\|_{1}^{2}\right)+\frac{1}{2}\left\|u^{j}-u^{j-1}\right\|_{1}^{2}+\Delta t\left\|u^{j}\right\|_{2}^{2} \\
& \quad \leq \Delta t C\left\|u^{j}\right\|_{1}^{2}+\left\langle\Delta W^{j}, u^{j}-u^{j-1}\right\rangle_{1}+\left\langle\Delta W^{j}, u^{j-1}\right\rangle_{1} .
\end{aligned}
$$

Thus, using a kick back argument on the second term, we obtain

$$
\begin{aligned}
\left\|u^{l}\right\|_{1}^{2} & +\sum_{j=1}^{l}\left\|u^{j}-u^{j-1}\right\|_{1}^{2}+\Delta t \sum_{j=1}^{l}\left\|u^{j}\right\|_{2}^{2} \\
& \leq C\left(\left\|u_{0}\right\|_{1}^{2}+\sum_{j=1}^{l}\left(\Delta t\left\|u^{j}\right\|_{1}^{2}+\left\|\Delta W^{j}\right\|_{1}^{2}+\left\langle\Delta W^{j}, u^{j-1}\right\rangle_{1}\right)\right) .
\end{aligned}
$$

Therefore,

$$
\begin{aligned}
& \mathbb{E} \sup _{l \in I_{N}}\left(\left\|u^{l}\right\|_{1}^{2}+\sum_{j=1}^{l}\left\|u^{j}-u^{j-1}\right\|_{1}^{2}+\Delta t \sum_{j=1}^{l}\left\|u^{j}\right\|_{2}^{2}\right) \\
& \leq C \mathbb{E}\left\|u_{0}\right\|_{1}^{2}+C \mathbb{E} \sup _{l \in I_{N}}\left(\sum_{j=1}^{l}\left(\Delta t\left\|u^{j}\right\|_{1}^{2}+\left\|\Delta W^{j}\right\|_{1}^{2}+\left\langle\Delta W^{j}, u^{j-1}\right\rangle_{1}\right)\right) \\
& \leq C \mathbb{E}\left\|u_{0}\right\|_{1}^{2}+C \mathbb{E}\left\{\sum_{j=1}^{N}\left(\Delta t\left\|u^{j}\right\|_{1}^{2}+\left\|\Delta W^{j}\right\|_{1}^{2}\right)\right\}+C \mathbb{E} \sup _{l \in I_{N}} \sum_{j=1}^{l}\left\langle\Delta W^{j}, u^{j-1}\right\rangle_{1} .
\end{aligned}
$$

Since $A^{1 / 2} \Delta W^{j}$ is a Gaussian random variable with covariance operator,

$$
\tilde{Q}:=\Delta t A^{1 / 2} Q^{1 / 2}\left(A^{1 / 2} Q^{1 / 2}\right)^{*},
$$

it follows, by (2.2), that

$$
\mathbb{E}\left\|\Delta W^{j}\right\|_{1}^{2}=\Delta t \operatorname{Tr} \tilde{Q}=\Delta t\left\|A^{1 / 2} Q^{1 / 2}\right\|_{\mathrm{HS}}^{2} .
$$

Next, note that $\sum_{j=1}^{l}\left\langle\Delta W^{j}, u^{j-1}\right\rangle_{1}$ is an Itô integral of the form $\int_{0}^{t_{l}}\left\langle\eta(t), \mathrm{d} A^{1 / 2} W(t)\right\rangle$, where $\eta$ is a piecewise continuous process, and, hence, also a martingale. Then using Hölder's 
inequality, the martingale inequality [7, Theorem 3.8], Itô's Isometry (2.5), (2.1), (2.2), and (4.1), we obtain

$$
\begin{aligned}
\left(\mathbb{E} \sup _{l \in I_{N}} \sum_{j=1}^{l}\left\langle\Delta W^{j}, u^{j-1}\right\rangle_{1}\right)^{2} & \leq \mathbb{E} \sup _{l \in I_{N}}\left(\sum_{j=1}^{l}\left\langle\Delta W^{j}, u^{j-1}\right\rangle_{1}\right)^{2} \\
& \leq 4 \sup _{l \in I_{N}} \mathbb{E}\left\{\sum_{j=1}^{l}\left\langle\Delta W^{j}, u^{j-1}\right\rangle_{1}\right\}^{2} \\
& =4 \mathbb{E} \Delta t \sum_{j=1}^{N}\left\|\tilde{Q}^{1 / 2} A^{1 / 2} u^{j-1}\right\|^{2} \\
& \leq 4\left\|\tilde{Q}^{1 / 2}\right\|^{2} \Delta t \sum_{j=1}^{N} \mathbb{E}\left\|u^{j-1}\right\|_{1}^{2} \\
& \leq 4\left\|\tilde{Q}^{1 / 2}\right\|_{\mathrm{HS}}^{2} \Delta t \sum_{j=1}^{N} \mathbb{E}\left\|u^{j-1}\right\|_{1}^{2} \\
& \leq C\left\|A^{1 / 2} Q^{1 / 2}\right\|_{\mathrm{HS}}^{2}\left(T+\mathbb{E}\left\|u_{0}\right\|^{2}+T\left\|Q^{1 / 2}\right\|_{\mathrm{HS}}^{2}\right) .
\end{aligned}
$$

Therefore, by (4.3), using also (4.1), we conclude that

$$
\mathbb{E} \sup _{l \in I_{N}}\left(\left\|u^{l}\right\|_{1}^{2}+\sum_{j=1}^{l}\left\|u^{j}-u^{j-1}\right\|_{1}^{2}+\Delta t \sum_{j=1}^{l}\left\|u^{j}\right\|_{2}^{2}\right) \leq C(1+T)
$$

and the proof is complete.

When proving strong convergence, even without rate, one needs bounds on higher moments of the time discretization. This will be achieved via a discrete Gronwall inequality, resulting in a bound that grows exponentially with time. However, since our approach does not provide rates for the strong error, this is not a major drawback. Note also, that this result is the exact time-discrete analogue of the bounds on the solution from Proposition 3.1.

Proposition 4.2. Let $p \geq 2, I_{n}=\{1,2, \ldots, n\}, 1 \leq n \leq N$, and $T=N \Delta t$. If $\left\|A^{1 / 2} Q^{1 / 2}\right\|_{\mathrm{HS}}$ $<\infty, \mathbb{E}\left\|u_{0}\right\|_{1}^{p}<\infty$, then there is $c=c(p, T, \beta)$ such that if $c \Delta t \leq \frac{1}{2}$, then

$$
\mathbb{E} \sup _{l \in I_{n}}\left\|u^{l}\right\|^{p}+\mathbb{E} \sup _{l \in I_{n}}\left\|u^{l}\right\|_{1}^{p} \leq C\left(T, p, u_{0}\right) .
$$

Proof. As noted in the proof of the previous proposition it is enough to bound the second term on the left-hand side. We start from (4.2) and take the $p$ th power of both sides for $p \geq 1$ to obtain

$$
\begin{gathered}
\left\|u^{l}\right\|_{1}^{2 p} \leq C\left(\left\|u_{0}\right\|_{1}^{2 p}+\left(\sum_{j=1}^{l} \Delta t\left\|u^{j}\right\|_{1}^{2}\right)^{p}+\left(\sum_{j=1}^{l}\left\|\Delta W^{j}\right\|_{1}^{2}\right)^{p}+\left(\sum_{j=1}^{l}\left\langle\Delta W^{j}, u^{j-1}\right\rangle_{1}\right)^{p}\right) \\
\leq C\left(\left\|u_{0}\right\|_{1}^{2 p}+\Delta t^{p-1} l^{p-1} \Delta t \sum_{j=1}^{l}\left\|u^{j}\right\|_{1}^{2 p}\right. \\
\left.+l^{p-1} \sum_{j=1}^{l}\left\|\Delta W^{j}\right\|_{1}^{2 p}+\left(\sum_{j=1}^{l}\left\langle\Delta W^{j}, u^{j-1}\right\rangle_{1}\right)^{p}\right) .
\end{gathered}
$$


Therefore,

$$
\begin{aligned}
& \mathbb{E} \sup _{l \in I_{n}}\left\|u^{l}\right\|_{1}^{2 p} \leq C\left(\mathbb{E}\left\|u_{0}\right\|_{1}^{2 p}+T^{p-1} \Delta t \sum_{j=1}^{n} \mathbb{E} \sup _{l \in I_{j}}\left\|u^{l}\right\|_{1}^{2 p}\right. \\
& \left.+n^{p-1} \sum_{j=1}^{n} \mathbb{E}\left\|\Delta W^{j}\right\|_{1}^{2 p}+\mathbb{E} \sup _{l \in I_{n}}\left(\sum_{j=1}^{l}\left\langle\Delta W^{j}, u^{j-1}\right\rangle_{1}\right)^{p}\right) .
\end{aligned}
$$

Next, we bound the last two terms in (4.4). We already noted that $A^{1 / 2} \Delta W^{j}$ is a Gaussian random variable with covariance operator $\tilde{Q}=\Delta t A^{1 / 2} Q^{1 / 2}\left(A^{1 / 2} Q^{1 / 2}\right)^{*}$. Hence, we use (2.2) and (2.7) to bound its $2 p$ th moment as

$$
\mathbb{E}\left\|\Delta W^{j}\right\|_{1}^{2 p} \leq C_{p}(\operatorname{Tr} \tilde{Q})^{p}=C_{p} \Delta t^{p}\left\|A^{1 / 2} Q^{1 / 2}\right\|_{\mathrm{HS}}^{2 p} .
$$

Therefore, it follows that

$$
n^{p-1} \sum_{j=1}^{n} \mathbb{E}\left\|\Delta W^{j}\right\|_{1}^{2 p} \leq C_{p} n^{p-1} \Delta t^{p} \sum_{j=1}^{n}\left\|A^{1 / 2} Q^{1 / 2}\right\|_{\mathrm{HS}}^{2 p} \leq C_{p} T^{p}\left\|A^{1 / 2} Q^{1 / 2}\right\|_{\mathrm{HS}}^{2 p} .
$$

For the last term in (4.4), we use the Burkholder-Davies-Gundy inequality (2.6), (2.1), and (2.2) to conclude that

$$
\begin{aligned}
\mathbb{E} \sup _{l \in I_{n}}\left(\sum_{j=1}^{l}\left\langle\Delta W^{j}, u^{j-1}\right\rangle_{1}\right)^{p} & \leq C_{p} \mathbb{E}\left\{\Delta t \sum_{j=1}^{n}\left\|\tilde{Q}^{1 / 2} A^{1 / 2} u^{j-1}\right\|^{2}\right\}^{p / 2} \\
& \leq C\left\|\tilde{Q}^{1 / 2}\right\|^{p} \Delta t^{p / 2} n^{p / 2-1} \sum_{j=1}^{n} \mathbb{E}\left\|u^{j-1}\right\|_{1}^{p} \\
\leq & C\left\|\tilde{Q}^{1 / 2}\right\|_{\mathrm{HS}}^{p} T^{p / 2-1} \Delta t \sum_{j=0}^{n-1}\left(\frac{1}{2}+\frac{1}{2} \mathbb{E} \sup _{l \in I_{j}}\left\|u^{l}\right\|_{1}^{2 p}\right) \\
& =C\left\|A^{1 / 2} Q^{1 / 2}\right\|_{\mathrm{HS}}^{p} T^{p / 2}+C\left\|A^{1 / 2} Q^{1 / 2}\right\|_{\mathrm{HS}}^{p} T^{p / 2-1} \Delta t \\
& \times \sum_{j=0}^{n-1}\left(\mathbb{E}_{l \in I_{j}} \operatorname{sul}_{l}^{l} \|_{1}^{2 p}\right) .
\end{aligned}
$$

Finally, substituting (4.5) and (4.6) into (4.4) yields the desired bound by using the discrete Gronwall inequality. Before applying the discrete Gronwall inequality, we use a kick back argument on the last term from the sum $T^{p-1} \Delta t \sum_{j=1}^{n} \mathbb{E} \sup _{l \in I_{j}}\left\|u^{l}\right\|_{1}^{2 p}$ in (4.4) using the condition $c \Delta t \leq \frac{1}{2}$.

\section{The convergence results}

We begin by showing a maximal type error estimate for the linear problem. Define the backward Euler approximation of the stochastic convolution $W_{A}\left(t_{n}\right)$ by

$$
W_{A}^{n}:=\sum_{k=1}^{n} E^{n-k+1} \Delta W^{k}=\sum_{k=1}^{n} \int_{t_{k-1}}^{t_{k}} E^{n-k+1} \mathrm{~d} W(s),
$$

where $E^{n}=(I+\Delta t A)^{-n}$. The following result has been proved in a wider generality for multiplicative noise in Banach spaces using heavy machinery in the range $0 \leq \beta<1$. This would 
be enough for the purposes of the semilinear problem with additive noise. However, it is possible to obtain the range $0 \leq \beta \leq 2$ because the noise is additive and the approximation of the noise is exact at the mesh points. Since this result is interesting on its own, and the proof presented here is rather elementary based on a discrete version of the factorization method, we present the result and the proof for the full range $0 \leq \beta \leq 2$.

Proposition 5.1. Let $\varepsilon \in\left(0, \frac{1}{2}\right), p>1 / \varepsilon, 0 \leq \beta \leq 2$, and $T=N \Delta t$. Then there is $C=C(p, \varepsilon, T)$ such that

$$
\left(\mathbb{E} \sup _{t_{n} \in[0, T]}\left\|W_{A}\left(t_{n}\right)-W_{A}^{n}\right\|^{p}\right)^{1 / p} \leq C \Delta t^{\beta / 2}\left\|A^{(\beta-1) / 2+\varepsilon} Q^{1 / 2}\right\|_{\mathrm{HS}}, \quad t_{n}=n \Delta t .
$$

Proof. Define the deterministic error operator $F_{n}$ by $F_{n}=E\left(t_{n}\right)-E^{n}$. It is well known that the following error estimate holds:

$$
\left\|A^{\rho / 2} F_{n} v\right\| \leq C \Delta t^{\beta / 2} t_{n}^{-(\beta-\gamma+\rho) / 2}\left\|A^{\gamma / 2} v\right\|, \quad 0 \leq \gamma \leq \beta+\rho, \rho, \gamma \geq 0, \beta \in[0,2] .
$$

Next, we consider the decomposition

$$
\begin{aligned}
W_{A}\left(t_{n}\right)-W_{A}^{n}= & \sum_{k=1}^{n} \int_{t_{k-1}}^{t_{k}}\left(E\left(t_{n}-\sigma\right)-E^{n-k+1}\right) \mathrm{d} W(\sigma) \\
= & \sum_{k=1}^{n} \int_{t_{k-1}}^{t_{k}}\left(E\left(t_{n}-\sigma\right)-E\left(t_{n-k+1}\right)\right) \mathrm{d} W(\sigma) \\
& +\sum_{k=1}^{n} \int_{t_{k-1}}^{t_{k}}\left(E\left(t_{n-k+1}\right)-E^{n-k+1}\right) \mathrm{d} W(\sigma) \\
= & : e_{1}^{n}+e_{2}^{n} .
\end{aligned}
$$

To estimate $e_{1}$, we first write

$$
e_{1}^{n}=\sum_{k=1}^{n} \int_{t_{k-1}}^{t_{k}} E\left(t_{n}-\sigma\right)\left(I-E\left(\sigma-t_{k-1}\right)\right) \mathrm{d} W(\sigma)=\int_{0}^{t_{n}} E\left(t_{n}-\sigma\right) \Psi(\sigma) \mathrm{d} W(\sigma)
$$

with $\Psi(\sigma)=\left(I-E\left(\sigma-t_{k-1}\right)\right)$ for $\sigma \in\left(t_{k-1}, t_{k}\right]$. Next, we use the factorization method from [7, Chapter 5] to write

$$
\begin{aligned}
e_{1}^{n} & =c_{\alpha} \int_{0}^{t_{n}} E\left(t_{n}-\sigma\right) \int_{\sigma}^{t_{n}}\left(t_{n}-s\right)^{-1+\alpha}(s-\sigma)^{-\alpha} \mathrm{d} s \mathrm{~d} W(\sigma) \\
& =c_{\alpha} \int_{0}^{t_{n}}\left(t_{n}-s\right)^{-1+\alpha} E\left(t_{n}-s\right) \int_{0}^{s}(s-\sigma) \Psi(\sigma) E(s-\sigma) \mathrm{d} W(\sigma) \mathrm{d} s \\
& =c_{\alpha} \int_{0}^{t_{n}}\left(t_{n}-s\right)^{-1+\alpha} E\left(t_{n}-s\right) Y(s) \mathrm{d} s,
\end{aligned}
$$

where $\alpha \in\left(0, \frac{1}{2}\right), c_{\alpha}^{-1}=\int_{\sigma}^{t}(t-s)^{-1+\alpha}(s-\sigma)^{-\alpha} \mathrm{d} s$ and

$$
Y(s)=\int_{0}^{s}(s-\sigma) \Psi(\sigma) E(s-\sigma) \mathrm{d} W(\sigma) .
$$


Therefore, by Hölder's inequality and $\|E(t)\| \leq 1$ for all $t \geq 0$,

$$
\mathbb{E} \sup _{t_{n} \in[0, T]}\left\|e_{2}^{n}\right\|^{p} \leq c_{\alpha}\left(\int_{0}^{T} s^{(-1+\alpha)(p /(p-1))} \mathrm{d} s\right)^{p-1} \int_{0}^{T} \mathbb{E}\|Y(s)\|^{p} \mathrm{~d} s .
$$

The first integral is finite for $p>1 / \alpha$. To bound the second integral, note that $Y(s)$ is a Gaussian random variable for all $s \in[0, T]$ and, therefore, we use (2.7) to bound its $p$ th moment, (2.1), (2.3) with $\beta=\frac{1}{2}-\varepsilon$, and (2.4) with $\beta=\gamma=0$ and $\rho=\beta / 2$, to obtain

$$
\begin{aligned}
\mathbb{E}\|Y(s)\|^{p} & \leq C_{p}\left(\int_{0}^{s}(s-\sigma)^{-2 \alpha}\left\|\Psi(\sigma) E(s-\sigma) Q^{1 / 2}\right\|_{\mathrm{HS}}^{2} \mathrm{~d} \sigma\right)^{p / 2} \\
& =C_{p}\left(\int_{0}^{s}(s-\sigma)^{-2 \alpha}\left\|\Psi(\sigma) A^{-\beta / 2} A^{1 / 2-\varepsilon} E(s-\sigma) A^{((\beta-1) / 2)+\varepsilon} Q^{1 / 2}\right\|_{\mathrm{HS}}^{2} \mathrm{~d} \sigma\right)^{p / 2} \\
& \leq C_{p}\left\|A^{((\beta-1) / 2)+\varepsilon} Q^{1 / 2}\right\|_{\mathrm{HS}}^{p}\left(\int_{0}^{s}(s-\sigma)^{-2 \alpha-1+2 \varepsilon}\left\|\Psi(\sigma) A^{-\beta / 2}\right\|^{2} \mathrm{~d} \sigma\right)^{p / 2} \\
& \leq C \Delta t^{\beta p / 2}\left\|A^{((\beta-1) / 2)+\varepsilon} Q^{1 / 2}\right\|_{\mathrm{HS}}^{p}\left(\int_{0}^{s}(s-\sigma)^{-2 \alpha-1+2 \varepsilon} \mathrm{d} s\right)^{p / 2} \\
& \leq C_{T, p, \alpha, \varepsilon} \Delta t^{\beta p / 2}\left\|A^{((\beta-1) / 2)+\varepsilon} Q^{1 / 2}\right\|_{\mathrm{HS}}^{p},
\end{aligned}
$$

provided that $\alpha<\varepsilon$. Given $p>1 / \varepsilon$, we thus need to choose $\alpha \in(1 / p, \varepsilon)$. We conclude with

$$
\int_{0}^{T} \mathbb{E}\|Y(s)\|^{p} \mathrm{~d} s \leq T C_{T, p, \alpha, \varepsilon} \Delta t^{\beta p / 2}\left\|A^{((\beta-1) 2)+\varepsilon} Q^{1 / 2}\right\|_{\mathrm{HS}}^{p},
$$

which proves the bound on $e_{1}^{n}$. To bound $e_{2}^{n}$, we use a discrete version of the factorization method. First introduce the constants

$$
c_{n, k}:=\left(\Delta t \sum_{l=k}^{n} t_{n-l+1}^{-1+\alpha} t_{l-k+1}^{-\alpha}\right)^{-1} .
$$

It is not difficult to see that $c_{n, k} \leq C$ for all $1 \leq k \leq n$. Then, we have

$$
\begin{aligned}
e_{2}^{n}= & \sum_{k=1}^{n} E\left(t_{n-k+1}\right) c_{n, k}\left(\Delta t \sum_{l=k}^{n} t_{n-l+1}^{-1+\alpha} t_{l-k+1}^{-\alpha}\right) \Delta W^{k} \\
& -\sum_{k=1}^{n} E^{n-k+1} c_{n, k}\left(\Delta t \sum_{l=k}^{n} t_{n-l+1}^{-1+\alpha} t_{l-k+1}^{-\alpha}\right) \Delta W^{k} \\
= & \Delta t \sum_{l=1}^{n} t_{n-l+1}^{-1+\alpha} E\left(t_{n-l}\right) \sum_{k=1}^{l} c_{n, k} t_{l-k+1}^{-\alpha} E\left(t_{l-k+1}\right) \Delta W^{k} \\
& -\Delta t \sum_{l=1}^{n} t_{n-l+1}^{-1+\alpha} E^{n-l} \sum_{k=1}^{l} c_{n, k} t_{l-k+1}^{-\alpha} E^{l-k+1} \Delta W^{k}
\end{aligned}
$$




$$
\begin{aligned}
& =\Delta t \sum_{l=1}^{n} t_{n-l+1}^{-1+\alpha} E\left(t_{n-l}\right) Y^{l}-\Delta t \sum_{l=1}^{n} t_{n-l+1}^{-1+\alpha} E^{n-l} \tilde{Y}^{l} \\
& =\Delta t \sum_{l=1}^{n} t_{n-l+1}^{-1+\alpha} F_{n-l} Y^{l}+\Delta t \sum_{l=1}^{n} t_{n-l+1}^{-1+\alpha} E^{n-l}\left(Y^{l}-\tilde{Y}^{l}\right) \\
& =: e_{21}^{n}+e_{22}^{n},
\end{aligned}
$$

where

$$
Y_{l}=\sum_{k=1}^{l} c_{n, k} t_{l-k+1}^{-\alpha} E\left(t_{l-k+1}\right) \Delta W^{k}, \quad \tilde{Y}_{l}=\sum_{k=1}^{l} c_{n, k} t_{l-k+1}^{-\alpha} E^{l-k+1} \Delta W^{k} .
$$

Next, we bound $e_{21}^{n}$, by Hölder's inequality and (5.1) with $\rho=0$ and $\gamma=\beta$, such that

$$
\begin{aligned}
\mathbb{E} \sup _{t_{n} \in[0, T]}\left\|e_{21}^{n}\right\|^{p} & \leq\left(\Delta t \sum_{l=1}^{N}\left(t_{l}^{-1+\alpha}\left\|F_{l} A^{-\beta / 2}\right\|\right)^{p /(p-1)}\right)^{p-1} \mathbb{E} \Delta t \sum_{l=1}^{N}\left\|A^{\beta / 2} Y^{l}\right\|^{p} \\
& \leq C \Delta t^{\beta p / 2}\left(\Delta t \sum_{l=1}^{n} t_{l}^{(-1+\alpha)(p /(p-1))}\right)^{p-1} \mathbb{E} \Delta t \sum_{l=1}^{N}\left\|A^{\beta / 2} Y^{l}\right\|^{p}
\end{aligned}
$$

where the first sum is finite if $p>1 / \alpha$. To estimate the last sum, note that $A^{\beta / 2} Y^{l}$ is a Gaussian random variable and, hence, as before, we use (2.7) to bound its $p$ th moment. Therefore, using also (2.1) and (2.3),

$$
\begin{aligned}
\mathbb{E} \Delta t \sum_{l=1}^{N}\left\|A^{\beta / 2} Y^{l}\right\|^{p} & =\Delta t \sum_{l=1}^{N} \mathbb{E}\left\|\left(\sum_{k=1}^{l} c_{n, k} t_{l-k+1}^{-\alpha} A^{\beta / 2} E\left(t_{l-k+1}\right) \Delta W^{k}\right)\right\|^{p} \\
& =\Delta t \sum_{l=1}^{N}\left(\Delta t \sum_{k=1}^{l} c_{n, k}^{2} t_{l-k+1}^{-2 \alpha}\left\|A^{\beta / 2} E\left(t_{l-k+1}\right) Q^{1 / 2}\right\|_{\mathrm{HS}}^{2}\right)^{p / 2} \\
& \leq C \Delta t \sum_{l=1}^{N}\left(\Delta t \sum_{k=1}^{N} t_{k}^{-2 \alpha}\left\|E\left(t_{k}\right) A^{1 / 2-\varepsilon} A^{((\beta-1) / 2)+\varepsilon} Q^{1 / 2}\right\|_{\mathrm{HS}}^{2}\right)^{p / 2} \\
& \leq C T\left\|A^{((\beta-1) / 2)+\varepsilon} Q^{1 / 2}\right\|_{\mathrm{HS}}^{p}\left(\Delta t \sum_{k=1}^{N} t_{k}^{-1-2 \alpha+2 \varepsilon}\right)^{p / 2} \\
& \leq C_{T, p, \alpha, \varepsilon}\left\|A^{((\beta-1) / 2)+\varepsilon} Q^{1 / 2}\right\|_{\mathrm{HS}}^{p},
\end{aligned}
$$

provided that $\alpha<\varepsilon$. Finally, we estimate $e_{22}^{n}$. By Hölder's inequality, we first obtain

$$
\begin{aligned}
\mathbb{E} \sup _{t_{n} \in[0, T]}\left\|e_{22}^{n}\right\|^{p} & \leq\left(\Delta t \sum_{l=1}^{N} t_{l}^{-1+\alpha}\left\|E^{l}\right\|^{p /(p-1)}\right)^{p-1} \mathbb{E} \sum_{l=1}^{N}\|Y(l)-\tilde{Y}(l)\|^{p} \\
& \leq\left(\Delta t \sum_{l=1}^{N} t_{l}^{(-1+\alpha)(p /(p-1))}\right)^{p-1} \mathbb{E} \sum_{l=1}^{N}\|Y(l)-\tilde{Y}(l)\|^{p} \\
& \leq C_{\alpha, p} \sum_{l=1}^{N}\|Y(l)-\tilde{Y}(l)\|^{p} \quad \text { if } p>\frac{1}{\alpha} .
\end{aligned}
$$


To estimate the last term, we use (2.7) to bound the $p$ th moment of a Gaussian random variable and also (2.1) and (5.1) with $\rho=1-2 \varepsilon$ and $\gamma=\beta$ to obtain

$$
\begin{aligned}
\sum_{l=1}^{N}\|Y(l)-\tilde{Y}(l)\|^{p} & =\sum_{l=1}^{N}\left\|\sum_{k=1}^{l} c_{n, k} t_{l-k+1}^{-\alpha} F_{l-k+1} \Delta W^{k}\right\|^{p} \\
& \leq C_{p} \sum_{l=1}^{N}\left(\Delta t \sum_{k=1}^{N} t_{k}^{-2 \alpha}\left\|F_{k} Q^{1 / 2}\right\|_{\mathrm{HS}}^{2}\right)^{p / 2} \\
& =C_{p} \sum_{l=1}^{N}\left(\Delta t \sum_{k=1}^{N} t_{k}^{-2 \alpha}\left\|A^{1 / 2-\varepsilon} F_{k} A^{-\beta / 2} A^{((\beta-1) / 2)+\varepsilon} Q^{1 / 2}\right\|_{\mathrm{HS}}^{2}\right)^{p / 2} \\
& \leq C_{p} \Delta t^{\beta p / 2}\left(\Delta t \sum_{k=1}^{N} t_{k}^{-1-2 \alpha+2 \varepsilon}\right)^{p / 2}\left\|A^{((\beta-1) / 2)+\varepsilon} Q^{1 / 2}\right\|_{\mathrm{HS}}^{p} \\
& \leq C_{T, p, \alpha, \varepsilon} \Delta t^{\beta p / 2}\left\|A^{((\beta-1) / 2)+\varepsilon} Q^{1 / 2}\right\|_{\mathrm{HS}}^{p},
\end{aligned}
$$

whenever $\alpha<\varepsilon$, which concludes the proof.

Next, we state a Lipschitz estimate for $f(u)$. Here, we use Sobolev's inequality and, similarly to (3.3), it is crucial that $d \leq 3$ and that the nonlinearity $f$ is at most cubic. For a proof; see [16, Lemma 2.5].

Lemma 5.1. For all $u, v \in \dot{H}^{1}$, we have

$$
\left\|A^{-1 / 2}(f(u)-f(v))\right\| \leq C\left(\|u\|_{1}^{2}+\|v\|_{1}^{2}\right)\|u-v\| .
$$

We are now ready to state and prove the pathwise convergence of the backward Euler scheme defined in (1.2).

Theorem 5.1. Let $\varepsilon>0,\left\|A^{1 / 2+\varepsilon} Q^{1 / 2}\right\|_{\mathrm{HS}}<\infty, 0 \leq \gamma<\frac{1}{2}$, and $T=N \Delta$ t. If $\mathbb{E}\left\|u_{0}\right\|_{1}^{2}<\infty$ then there are finite random variables $K \geq 0$ and $\Delta t_{0}>0$ such that, a.s.

$$
\sup _{t_{n} \in[0, T]}\left\|u\left(t_{n}\right)-u^{n}\right\| \leq K \Delta t^{\gamma}, \quad t_{n}=n \Delta t, \Delta t \leq \Delta t_{0} .
$$

Proof. Since the arguments are pathwise and, hence, basically deterministic, we omit standard details. Let $e_{n}=u\left(t_{n}\right)-u^{n}$ and $0 \leq \gamma<\frac{1}{2}$. We decompose the error, using the mild formulation of (1.2) and (3.2), as follows:

$$
\begin{aligned}
e^{n} & =\left(E\left(t_{n}\right) u_{0}-E^{n} u_{0}\right)+\left(W_{A}\left(t_{n}\right)-W_{A}^{n}\right)+\sum_{k=1}^{n} \int_{t_{k-1}}^{t_{k}} E\left(t_{n}-s\right) f(u(s))-E^{n-k+1} f\left(u^{k}\right) \mathrm{d} s \\
& =: e_{1}^{n}+e_{2}^{n}+e_{3}^{n} .
\end{aligned}
$$

By (5.1), we may estimate $e_{1}$ as

$$
\left\|e_{1}^{n}\right\| \leq C \Delta t^{1 / 2}\left\|u_{0}\right\|_{1} .
$$

For $e_{2}^{n}$, by Proposition 5.1 with $\beta=2$, we have

$$
\left\|e_{2}^{n}\right\| \leq L \Delta t\left\|A^{1 / 2+\varepsilon} Q^{1 / 2}\right\|_{\mathrm{HS}}
$$


a.s. for some finite nonnegative random variable $L$. Next, we can further decompose $e_{3}$ as

$$
\begin{aligned}
e_{3}^{n}= & \sum_{k=1}^{n} \int_{t_{k-1}}^{t_{k}} E^{n-k+1}\left(f\left(u\left(t_{k}\right)\right)-f\left(u^{k}\right)\right) \mathrm{d} s \\
& +\sum_{k=1}^{n} \int_{t_{k-1}}^{t_{k}}\left(E\left(t_{n-k+1}\right)-E^{n-k+1}\right) f\left(u\left(t_{k}\right)\right) \mathrm{d} s \\
& +\sum_{k=1}^{n} \int_{t_{k-1}}^{t_{k}} E\left(t_{n-k+1}\right)\left(f(u(s))-f\left(u\left(t_{k}\right)\right)\right) \mathrm{d} s \\
& +\sum_{k=1}^{n} \int_{t_{k-1}}^{t_{k}}\left(E\left(t_{n}-s\right)-E\left(t_{n-k+1}\right)\right) f(u(s)) \mathrm{d} s \\
= & e_{31}^{n}+e_{32}^{n}+e_{33}^{n}+e_{34}^{n} .
\end{aligned}
$$

To bound $e_{31}^{n}$, we use Propositions 3.1 and 4.1 together with Lemma 5.1 to conclude that for some finite nonnegative random variable $L_{1}$, we have a.s.

$$
\begin{aligned}
\left\|e_{31}^{n}\right\| & =\left\|\sum_{k=1}^{n} \int_{t_{k-1}}^{t_{k}} A^{1 / 2} E^{n-k+1} A^{-1 / 2}\left(f\left(u\left(t_{k}\right)\right)-f\left(u^{k}\right)\right) \mathrm{d} s\right\| \\
& \leq L_{1} \sum_{k=1}^{n} \int_{t_{k-1}}^{t_{k}} t_{n-k+1}^{-1 / 2}\left\|e^{k}\right\| \mathrm{d} s \\
& =L_{1} \Delta t \sum_{k=1}^{n} t_{n-k+1}^{-1 / 2}\left\|e^{k}\right\|,
\end{aligned}
$$

where we used the well-known fact that $\left\|A^{1 / 2} E^{k}\right\| \leq C t_{k}^{-1 / 2}$ (see, for example, [27, Lemma 7.3]). Next, we use Proposition 3.1, Lemma 5.1, and (5.1) with $\gamma=0, \rho=1$ and $\beta=2 \gamma$ to estimate $e_{32}^{n}$ as

$$
\begin{aligned}
\left\|e_{32}^{n}\right\| & =\left\|\sum_{k=1}^{n} \int_{t_{k-1}}^{t_{k}} A^{1 / 2}\left(E\left(t_{n-k+1}\right)-E^{n-k+1}\right) A^{-1 / 2} f\left(u\left(t_{k}\right)\right) \mathrm{d} s\right\| \\
& \leq \Delta t^{\gamma} L_{2} \sum_{k=1}^{n} \int_{t_{k-1}}^{t_{k}} t_{k}^{-1 / 2-\gamma} \mathrm{d} s \\
& =\Delta t^{\gamma} L_{2} \Delta t \sum_{k=1}^{n} t_{k}^{-1 / 2-\gamma}
\end{aligned}
$$

a.s. for some finite nonnegative random variable $L_{2}$. For $e_{33}^{n}$, we use the Hölder continuity of $u$ from Proposition 3.2 together with Proposition 3.1, Lemma 5.1, and (2.3) with $\beta=\frac{1}{2}$, and obtain

$$
\begin{aligned}
\left\|e_{33}^{n}\right\| & =\left\|\sum_{k=1}^{n} \int_{t_{k-1}}^{t_{k}} A^{1 / 2} E\left(t_{n-k+1}\right) A^{-1 / 2}\left(f(u(s))-f\left(u\left(t_{k}\right)\right)\right) \mathrm{d} s\right\| \\
& \leq \Delta t^{\gamma} L_{3} \sum_{k=1}^{n} \int_{t_{k-1}}^{t_{k}} t_{k}^{-1 / 2} \mathrm{~d} s \\
& =\Delta t^{\gamma} L_{3} \Delta t \sum_{k=1}^{n} t_{k}^{-1 / 2}
\end{aligned}
$$


a.s. for some finite nonnegative random variable $L_{3}$. Finally, by Proposition 3.1, Lemma 5.1, and (2.4) with $\beta=\frac{1}{2}$ and $\rho=0$, we have

$$
\begin{aligned}
\left\|e_{34}\right\| & =\left\|\sum_{k=1}^{n} \int_{t_{k-1}}^{t_{k}} A^{1 / 2}\left(E\left(t_{n}-s\right)-E\left(t_{n-k+1}\right)\right) A^{-1 / 2} f(u(s)) \mathrm{d} s\right\| \\
& \leq \Delta t^{\gamma} L_{4} \sum_{k=1}^{n} \int_{t_{k-1}}^{t_{k}} t_{k}^{-1 / 2-\gamma} \mathrm{d} s \\
& =\Delta t^{\gamma} L_{4} \Delta t \sum_{k=1}^{n} t_{k}^{-1 / 2-\gamma}
\end{aligned}
$$

a.s. for some finite nonnegative random variable $L_{2}$. Combining the estimates and using a generalized discrete Gronwall lemma [8, Lemma 7.1] concludes the proof.

Finally, we show strong convergence in $L^{p}$, albeit without rate.

Theorem 5.2. Let $\varepsilon>0, p \geq 1$, and $N \Delta t=T$. If $\left\|A^{1 / 2+\varepsilon} Q^{1 / 2}\right\|_{\mathrm{HS}}<\infty$ and $\mathbb{E}\left\|u_{0}\right\|_{1}^{q}<\infty$ for $q>p, q \geq 2$, then

$$
\lim _{\Delta t \rightarrow 0} \mathbb{E} \sup _{t_{n} \in[0, T]}\left\|u\left(t_{n}\right)-u^{n}\right\|^{p}=0, \quad t_{n}=n \Delta t .
$$

Proof. Let

$$
Y_{N}:=\sup _{t_{n} \in[0, T]}\left\|u\left(t_{n}\right)-u^{n}\right\|^{p}
$$

By Theorem 5.1 it follows that $Y_{N} \rightarrow 0$ a.s., and, hence, in probability, as $N \rightarrow \infty$. Let $s>1$ with $s p \leq q$. By Propositions 3.1 and 4.2 there is $M>0$ such that for $T^{q-1} \Delta t \leq \frac{1}{2}$,

$$
\mathbb{E} Y_{N}^{s} \leq C \mathbb{E} \sup _{t_{n} \in[0, T]}\left(\left\|u\left(t_{n}\right)\right\|^{s p}+\left\|u^{n}\right\|^{s p}\right) \leq M
$$

Therefore, it follows that $\left\{Y_{N}\right\}_{N \in \mathbb{N}}$ is uniformly integrable. Being convergent in probability and uniformly integrable, it converges in $L^{1}$; i.e.

$$
\lim _{\Delta t \rightarrow 0} \mathbb{E} \sup _{t_{n} \in[0, T]}\left\|u\left(t_{n}\right)-u^{n}\right\|^{p}=\lim _{N \rightarrow \infty} \mathbb{E} Y_{N}=0
$$

see [15, Proposition 3.12].

\section{References}

[1] Alabert, A. AND GyÖNGY, I. (2006). On numerical approximation of stochastic Burgers' equation. In From Stochastic Calculus to Mathematical Finance. Springer, Berlin, pp. 1-15.

[2] Blömker, D. And Jentzen, A. (2013). Galerkin approximations for the stochastic Burgers equation. SIAM J. Numer. Anal. 51, 694-715.

[3] Blömker, D., Kamrani, M. and Hosseini, S. M. (2013). Full discretization of the stochastic Burgers equation with correlated noise. IMA J. Numer. Anal. 33, 825-848.

[4] Cioica, P. et al. (2012). On the convergence analysis of Rothe's method. Preprint 124, DFG-Schwerpunktprogramm 1324

[5] Cox, S. And van NeErven, J. (2013). Pathwise Hölder convergence of the implicit-linear Euler scheme for semi-linear SPDEs with multiplicative noise. Numer. Math. 125, 259-345.

[6] Da Prato, G. and Debussche, A. (1996). Stochastic Cahn-Hilliard equation. Nonlinear Anal. 26, $241-263$. 
[7] Da Prato, G. And ZabczyK, J. (1992). Stochastic Equations in Infinite Dimensions (Encyclopedia Math. Appl. 44). Cambridge University Press.

[8] Elliott, C. M. ANd Larsson, S. (1992). Error estimates with smooth and nonsmooth data for a finite element method for the Cahn-Hilliard equation. Math. Comp. 58, 603-630, S33-S36.

[9] Gyöngy, I. And Millet, A. (2005). On discretization schemes for stochastic evolution equations. Potential Anal. 23, 99-134.

[10] Gyöngy, I. AND Millet, A. (2007). Rate of convergence of implicit approximations for stochastic evolution equations. In Stochastic Differential Equations: Theory and Applications. (Interdiscip. Math. Sci. 2), World Scientific, Hackensack, NJ, pp. 281-310.

[11] Hausenblas, E. (2002). Numerical analysis of semilinear stochastic evolution equations in Banach spaces. J. Comput. Appl. Math. 147, 485-516.

[12] Hausenblas, E. (2003). Approximation for semilinear stochastic evolution equations. Potential Anal. 18, 141-186.

[13] Higham, D. J., Mao, X. and Stuart, A. M. (2002). Strong convergence of Euler-type methods for nonlinear stochastic differential equations. SIAM J. Numer. Anal. 40, 1041-1063.

[14] Jentzen, A. (2009). Pathwise numerical approximation of SPDEs with additive noise under non-global Lipschitz coefficients. Potential Anal. 31, 375-404.

[15] Kallenberg, O. (1997). Foundations of Modern Probability. Springer, New York.

[16] Kovács, M., Larsson, S. And Mesforush, A. (2011). Finite element approximation of the Cahn-Hilliard-Cook equation. SIAM J. Numer. Anal. 49, 2407-2429.

[17] Kovács, M., Larsson, S. and Urban, K. (2013). On wavelet-Galerkin methods for semilinear parabolic equations with additive noise. In Monte Carlo and Quasi-Monte Carlo Methods 2012, Springer, Heidelberg, pp. 481-499.

[18] Kunita, H. (1990). Stochastic Flows and Stochastic Differential Equations (Camb. Stud. Adv. Math. 24). Cambridge University Press.

[19] LIU, D. (2003). Convergence of the spectral method for stochastic Ginzburg-Landau equation driven by spacetime white noise. Commun. Math. Sci. 1, 361-375.

[20] LIU, W. (2010). Invariance of subspaces under the solution flow of SPDE. Infin. Dimens. Anal. Quantum Prob. Relat. Top. 13, 87-98.

[21] LIU, W. (2013). Well-posedness of stochastic partial differential equations with Lyapunov condition. J. Differential Equat. 255, 572-592.

[22] LiU, W. ANd RöcKner, M. (2010). SPDE in Hilbert space with locally monotone coefficients. J. Funct. Anal. 259, 2902-2922.

[23] Pazy, A. (1983). Semigroups of Linear Operators and Applications to Partial Differential Equations (Appl. Math. Sci. 44). Springer, New York.

[24] Prévôt, C. And Röckner, M. (2007). A Concise Course on Stochastic Partial Differential Equations (Lecture Notes Math. 1905). Springer, Berlin.

[25] Printems, J. (2001). On the discretization in time of parabolic stochastic partial differential equations. $M 2 A N$ Math. Model. Numer. Anal. 35, 1055-1078.

[26] Sauer, M. and Stannat, W. (2015). Lattice approximation for stochastic reaction diffusion equations with one-sided Lipschitz condition. Math. Comp. 84, 743-766.

[27] Tноме́e, V. (2006). Galerkin Finite Element Methods for Parabolic Problems (Springer Ser. Comput. Math. 25), 2nd edn. Springer, Berlin. 